\title{
Polyhedral Properties of the $K$-median Problem on a Tree
}

\author{
Sven de Vries * \\ Marc E. Posner $\dagger$ \\ Rakesh V. Vohra ${ }^{\ddagger}$
}

* Zentrum Mathematik, TU München, D-80290 München, Germany.

Email: devries@mathematik.tu-muenchen.de

$\dagger$ Industrial Engineering, The Ohio State University, Columbus OH 43210, U.S.A.

Email: posner.1@osu.edu

$\ddagger$ Department of Managerial Economics and Decision Sciences, The Kellogg School,

Northwestern University, Evanston, ILL 60208, U.S.A.

Email: r-vohra@nwu.edu 


\begin{abstract}
The polyhedral structure of the K-median problem on a tree is examined. Even for very small connected graphs, we show that additional constraints are needed to describe the integer polytope. A complete description is given of those trees for which an optimal integer LP solution is guaranteed to exist. We present a new and simpler demonstration that an LP characterization of the 2-median problem is complete. Also, we provide a simpler proof of the value of a tight worst case bound for the LP relaxation. A new class of valid inequalities are identified. These inequalities are lifted to define facets for the K-median problem on a general graph. Also, we provide polyhedral descriptions for several types of trees. As part of this work, we summarize most known results for the K-median problem on a tree.
\end{abstract}

Key words and phrases: Polyhedral description, valid inequalities, facets, lifting 


\section{Introduction}

The $K$-median problem on a graph is a well known and much studied NP-hard problem (see Mirchandani [10]). To describe this problem, let $G=(V, E)$ be a connected graph where $V$ is the vertex set and $E$ is the edge set. For each $i, j \in V$, let $w_{i j}$ be the shortest distance between vertex $i$ and vertex $j$. We assume that $n=|V| \geq 3$ and that $w_{i j} \geq 0$ for all $i$ and $j$. The goal is to select $K$ vertices, called medians, so that the sum of the distances of each vertex to its nearest median is minimized. Let the decision variable

$$
x_{i j}= \begin{cases}1, & \text { if vertex } i \text { is assigned to a median at vertex } j \text { for } i, j \in V \\ 0, & \text { otherwise. }\end{cases}
$$

If $x_{j j}=1$, then a median is located at vertex $j \in V$. An integer programming formulation is

$$
\begin{aligned}
z^{*}=\min \sum_{i \in V} \sum_{j \in V} w_{i j} x_{i j} & \\
\text { sub. to } \quad \sum_{j \in V} x_{i j} & =1, \quad i \in V \\
x_{i j} & \leq x_{j j}, \quad i, j \in V \\
\sum_{j \in V} x_{j j} & =K \\
x_{i j} & \in\{0,1\}, \quad i, j \in V .
\end{aligned}
$$

While NP-hard in general, this problem is solvable in polynomial time if the underlying graph is a tree. The fastest known algorithm (in a worst case sense) takes $O\left(K n^{2}\right)$ steps and is due to Tamir [11]. In spite of this, no exact linear programming formulation of the $K$-median problem on trees is known.

The only sustained investigation of the $K$-median polytope is a 1986 dissertation by Lemke [8]. His thesis identifies some necessary and sufficient conditions for inequalities to be facets of the $K$-median problem. For the case where the problem is restricted to trees, he finds the dimension of the polytope. On the subject of the $K$-median problem restricted to trees, Lemke closes the dissertation on the following pessimistic note: 
"However, I found that merely finding the dimensionality of the polytopes to be sufficiently challenging to deter me from making a major effort to find the facets, although they may turn out to have a rather simple form."

In this paper, we survey what is known about the polyhedral structure of the $K$-median problem on trees. Little of this survey appears in published sources. Some general results appear in Section 2. In this section, we show that even for connected graphs on four vertices, additional constraints are needed to describe the integer polytope. Also, we characterize those graphs for which the LP polytope (the relaxation of the IP constraint set where $0 \leq$ $x_{i j} \leq 1$ for all $\left.i, j \in V\right)$ is either integral or has an optimal integer extreme point solution. In Section 3, a description of the 2-median problem is provided. We present a shorter and more direct proof than Goemans [6] that the description is complete. In Section 4, a new proof of the worst case bound of an LP solution is developed. This proof is simpler than the one found in Ward et al. [12]. In Section 5 we identify restrictions satisfied by at least one optimal solution. We determine the class of trees for which this restricted LP polytope is integral. A new set of valid inequalities are constructed in Section 6. We show that these inequalities are facets for the K-median problem on a general graph. In Section 7, these inequalities are used to find a description of a restricted integral polytope for trees called 2-stars. We conclude with some open issues.

\section{Properties of the Optimal Solution}

In this section, we show that the LP polytope is not integral even for graphs with only four vertices. Then, we examine two types of graphs that always have integral linear programming solutions. We show that for any other graph, there are cases when no integral linear programming solution exists. Finally, we find values of $K$ that guarantee the existence of an integral polytope. 
Unless otherwise specified, we assume that the connected underlying graph $G$ is a tree, $T$. Each vertex of the tree can be a median, and each vertex has a unit demand which must be satisfied by a median.

We define vertex $j$ to be a median if $x_{j j}>0$. A median where $x_{j j}=1$ is called integral, and a median where $0<x_{j j}<1$ is called fractional. We let $M \subseteq V$ be the set of medians and $I \subseteq M$ be the set of integral medians.

One difficulty in developing an exact linear programming representation of the $K$-median problem is incorporating the special structure of the objective function. Each $w_{i j}, i, j \in V$, in the objective function corresponds to a shortest path distance in the underlying tree. By incorporating additional path consistency constraints, researchers capture some of this structure. For $i, j \in V$, let $\tau(i, j)$ be the set of vertices on the unique path between $i$ and $j$, inclusively. Then, the path consistency constraints are

$$
x_{i j} \leq x_{p j}, \quad p \in \tau(i, j) \text { and } i, j \in V \text {. }
$$

Let $P(V)=P$ be the polytope defined by

$$
\begin{aligned}
\sum_{j \in V} x_{i j} & =1, \quad i \in V \\
x_{i j} & \leq x_{p j}, \quad p \in \tau(i, j), \quad i, j \in V \\
x & \geq 0 .
\end{aligned}
$$

It follows from a result of Barany et al. [3] that extreme points of $P$ are integral. The polytope $Q_{K}=P \cap\left\{x: \sum_{j \in V} x_{j j}=K\right\}$ defines the set of feasible solutions to (LP), the linear relaxation of the $K$-median problem. Unfortunately, even for small graphs, some extreme points are not integral.

Theorem 1 Every tree with at least four vertices has a data set where $Q_{K}$ has a fractional extreme point. 


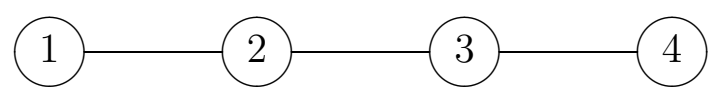

(a)

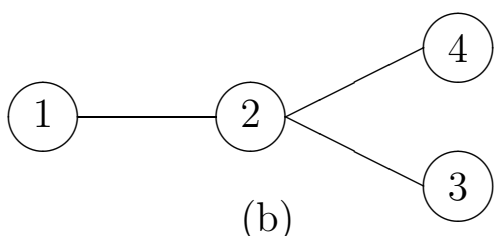

(b)

Figure 1: Graphs with fractional LP solutions for $K=2$.

Proof. Every tree with at least four vertices has one of the two graphs in Figure 1 as embedded subgraph.

For the case when $K=2$, consider the solution $x_{i i}^{0}=x_{i 2}^{0}=1 / 2$ for $i=1, \ldots 4, x_{23}^{0}=1 / 2$ and $x_{i j}^{0}=0$ otherwise. Suppose that $x^{0}$ is not an extreme point. Then, $x^{0}$ is the convex combination of two points $x^{1}, x^{2} \in Q_{K}$. This implies that $x_{i j}^{1}=x_{i j}^{2}=0$ whenever $x_{i j}^{0}=0$.

Because each vertex assigns the maximum amount to a median (if an assignment is made), it is not feasible to change $x_{i j}^{0}$ for $i \neq j$ without also changing the median assignments. Because vertices 1, 3 and 4 are assigned to 2, we cannot reduce $x_{22}^{0}$, and still maintain feasibility. Thus, $x_{22}^{1}=x_{22}^{2}=1 / 2$.

Suppose we try to increase $x_{i i}^{0}$ for some $i \in\{1,3,4\}$. To maintain feasibility, we must decrease $x_{22}^{0}$. However, we have established that this is not possible. Thus, $x^{0}$ is an extreme point solution.

To extend the embedded subgraph to a tree, for each additional vertex (over the four needed for the subgraph) increase $K$ by one and assign large distances to any new edges.

If

$$
w_{i j}= \begin{cases}1, & i=2, \quad j=3 \\ 2, & i=1,3,4, \quad j=2 \\ 10, & \text { otherwise },\end{cases}
$$

then the fractional extreme point solution given in the proof of Theorem 1 is the unique optimal solution. Observe that the values of $w$ are not symmetric and do not satisfy standard distance conditions. Thus, the characterization of the polytope for the K-median problem 
should include constraints that account for the fact that the distances between vertices are not arbitrary.

We say that $w>0$ has the distance property if $w_{i j}=w_{j i}$ and $w_{i j}=\sum_{(s, t) \in \tau(i, j)} w_{s t}$. Let $D$ be the set of $w$ 's with the distance property. We assume that $w \in D$ throughout this work.

The fractional solution associated with the path in Figure 1a occurs because assignments "skip" over fractional medians. Formally, skipping occurs whenever there are at least three vertices $i, p$ and $j$ such that $p \in \tau(i, j), x_{p p}, x_{j j}>0,0 \leq x_{i p}<x_{p p}$ and $x_{i j}>0$. Observe that this does not violate path consistency. However, when the solution is integral, path consistency prevents skipping. A solution $x$ has the no-skip property if $x_{i j}>0$ implies that $x_{i p}=x_{p p}$ for $i, j \in V$ and $p \in \tau(i, j)$.

Remark If $w \in D$, then an optimal solution to LP has the no-skip property.

Proof. Suppose that $p \in \tau(i, j), x_{i j}>0$ and $x_{i p}<x_{p p}$. Then, increasing $x_{i p}$ and reducing $x_{i j}$ by a suitably small $\epsilon>0$ reduces the solution value because $w_{i p}<w_{i j}$.

Given the values of $x_{j j}$ for each $j \in V$, we can determine a no-skip solution to (LP) (a fast procedure is described in Megiddo et. al. [9]). When $x_{j j} \in\{0,1\}$ for all $j$, then there is a no-skip solution to (IP). For vertex $i \in V$, let $j_{1}, j_{2}, \ldots, j_{n-1}$ be an ordering of the vertices in nondecreasing distance from $i$. Also, let $t$ be the smallest index such that $\sum_{k=1}^{t} x_{j_{k} j_{k}} \geq 1-x_{i i}$. Then,

$$
x_{i j_{t}}= \begin{cases}x_{j_{k} j_{k}}, & 1 \leq k \leq t-1 \\ 1-\sum_{k=1}^{t-1} x_{j_{k} j_{k}}, & k=t \\ 0, & k \geq t+1 .\end{cases}
$$

We now examine some properties of $Q_{K}$.

Property 1 (Ward et al. [12]) Every extreme point $x^{0}$ of $Q_{K}$ is either an extreme point of $P$ or the strict convex combination of two adjacent extreme points, $x^{A}$ and $x^{B}$, of $P$. If $x^{0}$ 
is not an extreme point of $P$, then $\left|\sum_{i \in V} x_{i i}^{0}-\sum_{i \in V} x_{i i}^{A}\right|>0$ and $\left|\sum_{i \in V} x_{i i}^{0}-\sum_{i \in V} x_{i i}^{B}\right|>0$. Further, if $K$ is integral, then $\left|\sum_{i \in V} x_{i i}^{B}-\sum_{i \in V} x_{i i}^{A}\right| \geq 2$.

Proof. Since $Q_{K}$ is the intersection of $P$ and a hyperplane, an extreme point of $Q_{K}$ must lie on an edge of $P$.

If $x^{0}$ is the strict convex combination of two adjacent points of $P$ and $\sum_{i \in V} x_{i i}^{0}=$ $\sum_{i \in V} x_{i i}^{A}=\sum_{i \in V} x_{i i}^{B}$, then $x^{0}$ is not an extreme point of $Q_{K}$.

When $K$ is integral, so is $\sum_{i \in V} x_{i i}^{0}$. By assumption, $x^{0}=\lambda x^{A}+(1-\lambda) x^{B}$ for $\lambda \in$ $(0,1)$, and $\sum_{i \in V} x_{i i}^{A}$ and $\sum_{i \in V} x_{i i}^{B}$ are integral. Observe that $\sum_{i \in V} x_{i i}^{0}$ cannot be integral if $\left|\sum_{i \in V} x_{i i}^{B}-\sum_{i \in V} x_{i i}^{A}\right| \leq 1$

Define $\bar{J}$ to be the complement of set $J$.

Property 2 Suppose an extreme point $x^{0} \in Q_{K}$ has the set of integral medians $I$. Let tree $T^{\prime}=\left(V \backslash I, E^{\prime}\right)$, where $(i, j) \in E^{\prime}$ if $x_{i j}^{0}>0$ or $x_{j i}^{0}>0$, for $i, j \in V \backslash I$. Then, at most one connected component of $T^{\prime}$ contains fractional medians.

Proof. Suppose that $T^{\prime}$ has two connected components with vertex sets $V_{1}$ and $V_{2}$, where each component contains fractional medians. For $i=1,2$, let $J_{i}=V_{i} \cup\left\{k \in I: x_{j k}^{0}>\right.$ 0 for some $\left.j \in V_{i}\right\}$ and let $K_{i}=\sum_{j \in J_{i}} x_{j j}^{0}$. Since $x^{0}$ is an extreme point of $Q_{K}, x^{0}$ restricted to $J_{i}$, denoted by $x_{J_{i}}^{0}$, is an extreme point of $Q_{K_{i}}\left(J_{i}\right) \cap\left\{x: x_{k k}=1\right.$ for $\left.k \in I\right\}$. Furthermore, $x_{J_{i}}^{0}$ is fractional.

Polytope $P\left(J_{i}\right)$ is integral, for $i=1,2$. Hence, $P\left(J_{i}\right) \cap\left\{x: x_{k k}=1\right.$ for $\left.k \in I\right\}$ is also integral. Now, Property 1 implies that $x_{J_{i}}^{0}$ is a convex combination of two extreme points of $P\left(J_{i}\right) \cap\left\{k \in I: x_{k k}=1\right\}$ for $i=1,2$. As a result, for some $\epsilon>0$, there are points (not necessarily extremal) $x_{J_{i}}^{1} \in Q_{K_{i}+\epsilon}\left(J_{i}\right) \cap\left\{x: x_{k k}=1\right.$ for $\left.k \in I\right\}$ and $x_{J_{i}}^{2} \in$ $Q_{K_{i}-\epsilon}\left(J_{i}\right) \cap\left\{x: x_{k k}=1\right.$ for $\left.k \in I\right\}$ where $x_{J_{i}}^{1} / 2+x_{J_{i}}^{2} / 2=x_{J_{i}}^{0}$. Define $\oplus$ to be the operator that concatenates two vectors with $s_{1}$ and $s_{2}$ components, respectively, into one vector with 
$s_{1}+s_{2}$ components. Let $x^{a}=x_{V_{1}}^{1} \oplus x_{V_{2}}^{2} \oplus x_{V_{1} \cup V_{2}}^{0}$ and $x^{b}=x_{V_{1}}^{2} \oplus x_{V_{2}}^{1} \oplus x_{\overline{V_{1} \cup V_{2}}}^{0}$. Since $x^{a}, x^{b} \in Q_{K}$ and $x^{a} / 2+x^{b} / 2=x^{0}$, we have that $x^{0}$ is not an extreme point of $Q_{K}$. Contradiction.

Although Theorem 1 establishes that $Q_{K}$ may not be integral, there are two types of graphs where an optimal integral LP solution exists. While the next result appears to be well known, a proof is provided because we have not found one in the literature. Let $z^{L P}$ be the optimal value of the LP solution.

Theorem 2 If $G$ is a path, then $z^{L P}=z^{*}$.

Proof. Assume that the vertices of the path are numbered in increasing order. Suppose an extreme point solution $x^{0}$ to (LP) has some fractional medians. From Property 2, the graph induced by the set of fractional medians is exactly one line segment. From Property 1 , $x^{0}$ is a convex combination of two integral extreme points of $P, x^{A}$ and $x^{B}$. Hence, $x^{0}=$ $\lambda x^{A}+(1-\lambda) x^{B}$ where $0<\lambda<1$. Let $A=\left\{j: x_{j}^{A}=1\right\}$ and $B=\left\{j: x_{j}^{B}=1\right\}$.

If the membership of the fractional vertices along the line segment alternate between sets $A$ and $B$, then $\| A|-| B|| \leq 1$. This violates Property 1 . Thus, some pair of adjacent medians, $i_{1}$ and $i_{2}$, where $i_{1}<i_{2}$ belong to the same set. Without loss of generality, let this set be $B$.

Because $x^{B}$ is integral, there is some vertex $q \in\left[i_{1}, i_{2}\right]$ such that $x_{j i_{1}}^{B}=1$ for $j \in \tau\left(i_{1}, q\right)$ and $x_{j i_{2}}^{B}=1$ for $j \in \tau\left(q+1, i_{2}\right)$. Now, suppose that $x_{j k}^{A}>0$ for some $j \in\left[i_{1}, q\right]$ and $k>i_{2}$. Since $x^{0}=\lambda x^{A}+(1-\lambda) x^{B}$, we have that $x_{j i_{2}}^{0}=0$. However, $x_{j k}^{0}>0$ contradicts the no-skip property. A similar argument applies if $x_{j k}^{A}>0$ for $j \in\left[q+1, i_{2}\right]$ and $k<i_{1}$. Thus, $x_{j k}^{A}=0$ for $j \leq q$ and $k \geq q$, and for $j \geq q+1$ and $k \leq q$. Consequently, the solution corresponding to $x^{0}$ has two disconnected components, both of which have fractional medians. However, this contradicts Property 2.

For an alternative demonstration of Theorem 2, Kolen and Tamir [7] present an extended formulation for the k-median problem on a tree. When the tree is a line graph, the constraint 
matrix is totally unimodular. This implies that the polytope corresponding to the extended formulation is integral. While projecting out the extended variables is non-trivial, the $\mathrm{k}$ median polytope must also be integral.

Now, consider a tree where only the center vertex, $c$, has degree $d \geq 3$. Each path from the center vertex to a leaf node contains at most $v=(n-1) / d$ vertices. We call this graph a $v$-star. Figure $1 \mathrm{~b}$ is an example of a 1-star and Figure 4 in Section 6 is an example of a 2-star.

Theorem 3 If $G$ is a 1-star, then $z^{L P}=z^{*}$.

Proof. Let $x^{*}$ be an optimal fractional solution to LP. Suppose that $x_{c c}^{*}=1$. Then Property 2 establishes that only one leaf node has a fractional value. This implies that $K$ is not integral. Contradiction.

As a result, we assume that $x_{c c}^{*}<1$ for every optimal solution to LP. Consequently, for some $q \in V \backslash\{c\}$, we have that $x_{c q}^{*}>0$. Consider the new solution $x^{\prime}$ where

$$
x_{j j}^{\prime}= \begin{cases}x_{c c}^{*}+x_{c q}^{*}, & j=c \\ x_{q q}^{*}-x_{c q}^{*}, & j=q \\ x_{j j}^{*}, & j \neq c, q,\end{cases}
$$

and all other assignments are made according to (1). Since $w_{c q}=w_{q c}$, this new solution is optimal. Repeat this process with other leaf nodes until the median value of $c$ is 1 . The procedure terminates because one fractional median is removed at each step. This contradicts the assumption that $x_{c c}^{*}<1$.

We provide the negative result that for any other type of tree, all optimal linear programming solution may be fractional.

Theorem 4 The only trees where $z^{L P}=z^{*}$ for all values of $K$ and all $w \in D$ are paths and 1-stars. 


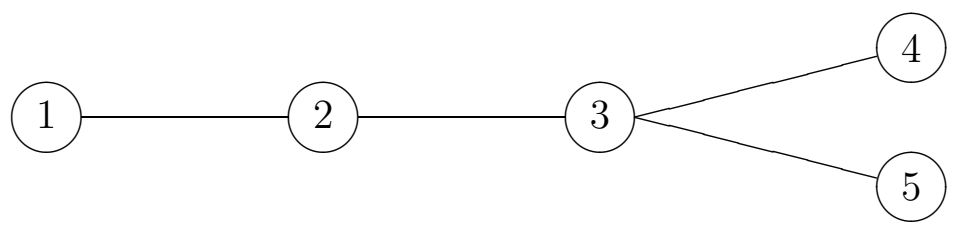

Figure 2: Graph with fractional LP solution for $K=2$.

Proof. Consider the graph in Figure 2. This graph is embedded in each tree which is not a path or a 1-star. When $K=2$ and the arc distances are $w_{12}=2, w_{23}=1$, and $w_{34}=w_{35}=4$, the unique optimal solution to (LP) is $x_{j j}^{L P}=1 / 2$ for $j=2,3,4,5$ and all other assignments made according to (1). Hence, by an appropriate choice of arc distances (set distances to a suitably small $\epsilon>0$ for all arcs not part of the embedded subgraph) and increase $K$ by the number of additional vertices, we can construct a nonintegral extreme point.

For a given $K$, we now describe necessary and sufficient conditions for the LP polytope to be integral for a general graph.

Theorem 5 If $K \in\{1, n-1, n\}$, then $Q_{K}$ is integral. There exist counter-examples for any $K \in\{2,3, \ldots, n-2\}$.

Proof. When $K=1$, an extreme point solution cannot be a convex combination of extreme points of $P$. Consequently, from Property 1, the solution must be integral.

Suppose $K=n-1$. If there is a fractional extreme point solution $x^{0}$, then it follows from Property 1 , that there are sets $A$ and $B$, and $\lambda \in(0,1)$, where $|A| \leq n-2,|B|=n$, and $x^{0}=\lambda x^{A}+(1-\lambda) x^{B}$. Consequently, $x_{i i}^{B}=1$ for all $i \in V$. Since $x^{0}$ is fractional, there are at least two fractional medians. Because the medians must be in the same component by Property 2 , there are vertices $j, k \in V$ such that $0<x_{j k}^{0}<1$ and $0<x_{k k}^{0}<1$. This implies that $x_{j j}^{A}=x_{k k}^{A}=0$. Hence, $x_{j k}^{A}=0$. Since $x_{j k}^{B}=0$, it is not possible that $x_{j k}^{0}>0$. Contradiction. 


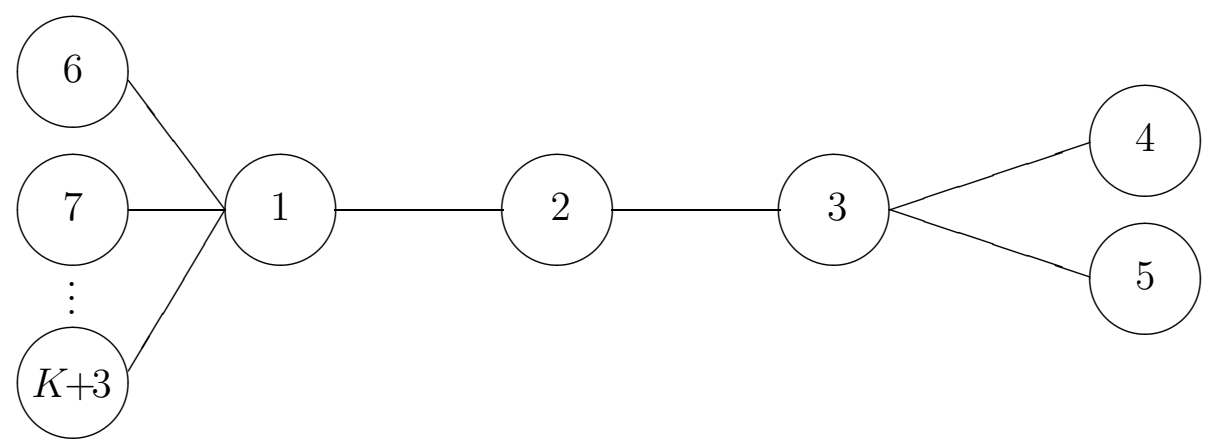

Figure 3: Graph with fractional LP solution for $K \notin\{1, n-1, n\}$.

When $K=n$, the only solution is to have an integral median at every vertex.

For any other value of $K$, consider the graph shown in Figure 3. The distances on the arcs are $w_{12}=2, w_{23}=1, w_{35}=w_{45}=4$, and $w_{i 1}=100$ for $i=6,7, \ldots, K+3$. From the proof of Theorem 4 , the embedded graph with vertices $\{1,2, \ldots, 5\}$ has a fractional LP solution.

\section{When $K=2$}

Lemke [8] refers to an unpublished manuscript for a proof that the following is a complete linear description of the 2-median polytope $\left(\mathrm{LP}_{2}\right)$ :

$$
\begin{aligned}
\sum_{j \in V} x_{i j} & =1, \quad i \in V \\
\sum_{i \in V}\left(d_{i}-2\right) x_{i j}+x_{j j} & =0, \quad j \in V \\
x_{i j} & \leq x_{k j}, \quad i, j \in V, k \in \tau(i, j),
\end{aligned}
$$

where $d_{i}$ is the degree of vertex $i$.

Independently, Goemans [6] establishes that the above formulation describes an integer polytope. His proof uses an extended formulation of the 2-median problem. Then, the auxiliary variables are projected out. In this section, we provide a simpler proof as well as show that both the cardinality constraint $\sum_{i \in V} x_{i i}=2$ and the non-negativity constraints are redundant. 
Theorem 6 Any solution $x$ to $\left(L P_{2}\right)$ satisfies $\sum_{j \in V} x_{j j}=2$.

Proof. Summing (2) over all $j \in V$ gives

$$
\begin{aligned}
\sum_{j \in V} x_{j j} & =-\sum_{j \in V} \sum_{i \in V}\left(d_{i}-2\right) x_{i j} \\
& =-\sum_{i \in V}\left(d_{i}-2\right)\left[\sum_{j \in V} x_{i j}\right] \\
& =-\sum_{i \in V}\left(d_{i}-2\right) \\
& =2 .
\end{aligned}
$$

The last equality holds because $\sum_{i \in V} d_{i}=2(n-1)$ when the graph is a tree. Thus, (2) implies the cardinality constraint.

Theorem 7 Any solution $x$ to $\left(L P_{2}\right)$ is non-negative.

Proof. To show that the non-negativity constraints are redundant, we establish that $x_{l p} \geq 0$ for every vertex $p$ and leaf vertex $l$. Non-negativity of all other variables follows by path consistency. Let $L$ be the set of leaves of tree $T$. We consider two cases: $p \in L$, and $p \notin L$. Case 1. $p \in L$.

Let $l \in L$ and $l \neq p$. Since $d_{l}=d_{p}=1$,

$$
\sum_{i \in V}\left(d_{i}-2\right) x_{i p}+x_{p p}=\sum_{i \in V \backslash L}\left(d_{i}-2\right) x_{i p}-\sum_{j \in L \backslash\{l, p\}} x_{j p}-x_{l p} .
$$

We first establish that $\sum_{i \in V \backslash L}\left(d_{i}-2\right) x_{i p}-\sum_{j \in L \backslash\{l, p\}} x_{j p} \geq 0$. Let $H \subseteq T$ be the graph of the unique path from $l$ to $p$, and let $V_{H}$ be the associated vertex set. Let $d_{j}^{H}$ be the degree of vertex $j \in V_{H}$ in $H$. Because $H$ is a path, $d_{j}^{H}=2$ for $j \in V_{H} \backslash L$. Since there are no leaf nodes in $H$ other than $l$ and $p, \sum_{i \in V_{H} \backslash L}\left(d_{i}^{H}-2\right) x_{i p}-\sum_{j \in\left(V_{H} \cap L\right) \backslash\{l, p\}} x_{j p} \geq 0$.

Now, select $k \in L \backslash V_{H}$. Consider the unique path $\tau(k, p)$ from $k$ to $p$. Let $q$ be the first vertex in $H$ reached on this path. With the exception of $q$ and $k, d_{i}^{\tau(k, q)}=2$ for each vertex $i$ on the path $\tau(k, q)$. The degree of $k$ is 1 and $d_{q}^{H} \geq 2$. Let $H^{\prime}=H \cup \tau(k, q)$. Since $x_{q p} \geq x_{k p}$ 
by path consistency,

$$
\begin{aligned}
\sum_{i \in V_{H^{\prime}} \backslash L}\left(d_{i}^{H^{\prime}}-2\right) & x_{i p}-\sum_{j \in\left(V_{H^{\prime}} \cap L\right) \backslash\{l, p\}} x_{j p} \\
& =\sum_{i \in V_{H} \backslash L}\left(d_{i}^{H}-2\right) x_{i p}-\sum_{j \in\left(V_{H} \cap L\right) \backslash\{l, p\}} x_{j p}+\left[\left(d_{q}^{H}+1-2\right)-\left(d_{q}^{H}-2\right)\right] x_{q p}-x_{k p} \\
& \geq 0 .
\end{aligned}
$$

Let $H=H^{\prime}$, and repeat the process. When $L \backslash V_{H}=\emptyset$, we have that $H=T$ and $\sum_{i \in V \backslash L}\left(d_{i}-2\right) x_{i p}-\sum_{j \in L \backslash\{l, p\}} x_{j p} \geq 0$. From (3), $-x_{l p} \leq 0$, and non-negativity of the variables is established for this case.

Case 2. $p \notin L$.

Select $k \in L$ such that $p$ is on the path $\tau(k, l)$. Since $d_{k}=d_{l}=1$,

$$
\sum_{i \in V}\left(d_{i}-2\right) x_{i p}+x_{p p}=0=\sum_{i \in V \backslash L}\left(d_{i}-2\right) x_{i p}-\sum_{j \in L \backslash\{k, l\}} x_{j p}+x_{p p}-x_{k p}-x_{l p} .
$$

Similar to Case 1, we can show that $\sum_{i \in V \backslash L}\left(d_{i}-2\right) x_{i p}-\sum_{j \in L \backslash\{k, l\}} x_{j p} \geq 0$. From (4), $x_{p p}-x_{k p}-x_{l p} \leq 0$. Since $x_{p p} \geq x_{k p}$, we have that $-x_{l p} \leq 0$. Thus, non-negativity of the variables is implied by the constraints of $\left(\mathrm{LP}_{2}\right)$.

We show that $\left(\mathrm{LP}_{2}\right)$ is integral.

Theorem 8 If $x^{0}$ is an optimal extreme point solution of $\left(L P_{2}\right)$, then $x^{0}$ is integral.

Proof. Consider an extreme point solution $x^{0}$ to (2) and suppose it is fractional. Then, $x^{0}$ can be expressed as a convex combination of integral extreme points of $(\mathrm{P})$, i.e. $x^{0}=$ $\sum_{r=1}^{t} \mu_{r} x^{r}$

For each $p \in M$ and each $r$, let $N_{p}^{r}=\left\{i \in V: x_{i p}^{r}=1\right\}$. Let $N_{p}^{r}$ be the vertex set of graph $H_{p}^{r}$. If $i, j \in N_{p}^{r}$ and $(i, j)$ is an edge in tree $T$, then let $(i, j)$ be an edge in $H_{p}^{r}$. Graph $H_{p}^{r}$ is a tree because path consistency implies that $H_{p}^{r}$ is connected. 
Denote the number of edges of $T$ with exactly one endpoint in $N_{p}^{r}$ by $\delta\left(N_{p}^{r}\right)$. Since $H_{p}^{r}$ is a tree and since the sum of the degrees of the nodes in a tree is $2 \times($ number of nodes -1$)$,

$$
\delta\left(N_{p}^{r}\right)=\sum_{i \in N_{p}^{r}} d_{i}-2\left(\left|N_{p}^{r}\right|-1\right)=\sum_{i \in N_{p}^{r}}\left(d_{i}-2\right)+2 .
$$

From (2),

$$
\begin{aligned}
0 & =\sum_{i \in V}\left(d_{i}-2\right) x_{i q}^{0}+x_{p p}^{0} \\
& =\sum_{r=1}^{t} \mu_{r}\left[\sum_{i \in V}\left(d_{i}-2\right) x_{i p}^{r}+x_{p p}^{r}\right] \\
& =\sum_{r=1}^{t} \mu_{r}\left[\delta\left(N_{p}^{r}\right)-1\right] x_{p p}^{r}
\end{aligned}
$$

The last term follows from (5) and the integrality of $x^{r}$.

Let $K^{r}=\sum_{j \in V} x_{j j}^{r}$. Then, $\sum_{r=1}^{t} \mu_{r} K^{r}=2$. Given an integral solution $x^{r}$, for each $p$ such that $x_{p p}^{r}=1$, contract the vertices in $N_{p}^{r}$ into a single vertex. The result is a tree with one vertex corresponding to each $p$ such that $x_{p p}^{r}=1$. Further, the degree of this vertex is $\delta\left(N_{p}^{r}\right)$. Consequently, $\delta\left(N_{j}^{r}\right) \leq K^{r}-1$ for all $j \in V$.

Since $x^{0}$ is fractional, there is an $r$, call it $r^{\prime}$, such that $K^{r^{\prime}} \geq 3$. Otherwise, $x^{0}$ is a convex combination of 2-median solutions and is not an extreme point. Because every tree contains at least one leaf node, there must be a $q \in V$ such that $x_{q q}^{r}=1$ and $\delta\left(N_{p}^{r}\right)=1$. Consequently, for $r^{\prime}$, there is a $q \in V$ such that $x_{q q}^{r^{\prime}}=1$ and $\delta\left(N_{q}^{r^{\prime}}\right)<K^{r^{\prime}}-1$. Now, (6) gives

$$
\begin{aligned}
0 & =\sum_{r=1}^{t} \mu_{r}\left[\delta\left(N_{q}^{r}\right)-1\right] x_{q q}^{r} \\
& =\sum_{r \neq r^{\prime}} \mu_{r}\left[\delta\left(N_{q}^{r}\right)-1\right] x_{q q}^{r}+\mu_{r^{\prime}}\left[\delta\left(N_{q}^{r^{\prime}}\right)-1\right] \\
& <\sum_{r \neq r^{\prime}} \mu_{r}\left[K^{r}-2\right]+\mu_{r^{\prime}}\left[K^{r^{\prime}}-2\right] \\
& =\sum_{r=1}^{t} \mu_{r} K^{r}-2 \sum_{r=1}^{t} \mu_{r} \\
& =0
\end{aligned}
$$

This contradiction establishes the result. 


\section{Strength of the LP Relaxation}

When the constraint that $\sum_{j=1}^{n} x_{i j}=1$ is relaxed for one or more $i \in V$, Ward et al. [12] show that

$$
z^{*} / z^{L P} \leq 2-2 /(K+1)
$$

They also show that this bound is tight. The proof involves the analysis of an algorithm for finding a special kind of vertex cover. Their proof can be extended to (IP). In this section, we present a shorter proof for (IP) by replacing the algorithm with an induction argument. Analogous results for general graphs can be found in Arya et al. [1].

Given a set of medians $M$, let

$$
z(M)=\sum_{i \in V} \min _{j \in M}\left\{w_{i j}\right\}
$$

Hence, the K-median problem can be expressed as $\min _{M \subseteq V}\{z(M):|M|=K\}$.

From Property 1, associated with each fractional extreme point solution of $Q_{K}$ are two sets $A, B \subseteq V$ and $\lambda \in(0,1)$ such that

$$
\begin{aligned}
& |A|<K<|B| \\
& z(A) \geq z^{L P} \geq z(B) \\
& z^{L P}=\lambda z(A)+(1-\lambda) z(B) \\
& K=\lambda|A|+(1-\lambda)|B| .
\end{aligned}
$$

We construct a set $S \subseteq A \cup B$ with cardinality $K$ which satisfies (7) with equality. The proof of (7) is based on Lemmas 1 and 2. Theorem 9 as well as the statement of Lemma 2 are found in Ward et al. [12].

Let $F(A, B)$ be a forest, where $A, B$ partitions the vertices of $F$ so that adjacent vertices are in different sets. Without loss of generality, assume that $a=|A| \leq b=|B|$. A K-cover 
is defined to be a set of $K$ vertices of a given graph such that every arc is incident to at least one element of the set.

Lemma 1 Suppose that every vertex $v \in A$ of $F(A, B)$ has degree at least three. Then, for any integer $K$ where $a \leq K \leq b$, there is a $K$-cover $S$ such that $|S \cap B| \geq 2 K-b$.

Proof. If $K=b$, then $S=B$ satisfies the conditions of the lemma. As a result, we only consider the case when $K<b$. The remainder of the proof is by induction on $d=b-a$. When $d=0$, the lemma is true because $S=B$ is the required cover.

Now, let $F(A, B)$ be a graph with the smallest $d \geq 1$ for which the lemma is false. First we show that there is at least one vertex $p \in A$ which has at least two neighbors that are leaves. Since all vertices of $A$ have degree at least three, all leaves of $F(A, B)$ are elements of $B$. Delete all the leaves of $F(A, B)$ and their incident edges. In this reduced tree, no vertex in $B$ is a leaf. Consequently, at least one vertex, $p$, in $A$ must now be a leaf. Let $L_{p}$ be the neighbors of $p$ that are leaves in $F(A, B)$. Since all vertices in $A$ have degree at least three, $\left|L_{p}\right| \geq 2$ and at least two neighbors of $p$ are leaves.

Construct $F\left(A^{\prime}, B^{\prime}\right)$ by deleting $p$, two vertices of $L_{p}$, and the incident edges. Thus, $\left|A^{\prime}\right|=a-1$ and $\left|B^{\prime}\right|=b-2$. Also, these changes do not alter the degree of the vertices in $A \backslash\{p\}$. Hence, $F\left(A^{\prime}, B^{\prime}\right)$ satisfies the conditions of the lemma. Since $K<b$, the induction hypothesis implies that there is a $K-1$ cover $S^{\prime}$ for $F\left(A^{\prime}, B^{\prime}\right)$ such that $\left|S^{\prime} \cap B^{\prime}\right| \geq$ $2(K-1)-(b-2)=2 K-b$. However, $S=S^{\prime} \cup\{p\}$ is a $K$ cover for $F(A, B)$ where $|S \cap B| \geq 2 K-b$. Contradiction.

Consider a set of real valued weights $u_{j}, j \in B$, such that $\sum_{j \in B} u_{j} \geq 0$. Let $U=\sum_{j \in B} u_{j}$.

Lemma 2 Suppose we are given $F(A, B)$, integer $K$ for $a \leq K \leq b$, and real valued weights $u_{j}$ such that $j \in B$. Then, there is a vertex cover $S$ of cardinality $K$, such that

$$
(2 b-K-a) \sum_{j \in B \cap S} u_{j} \geq(K-a) U
$$


Proof. If $a=K$, then $S=A$ satisfies the conditions of the lemma. Also, if $b=K$, then $S=B$ satisfies the conditions of the lemma. Consequently, we consider the case where $a<K<b$.

The remainder of the proof is by induction on the number of vertices in $F(A, B)$. When $F(A, B)$ has two vertices, then one is in $A$ and the other is in $B$. The result is established by observing that $a=b=K$.

Now, let $F(A, B)$ be a graph with the fewest number of vertices that is a counterexample to the lemma. Suppose that $F(A, B)$ has a vertex $p \in A$, where $p$ has degree one or two. When $p$ has degree one, let $v_{1} \in B$ be the neighbor of $p$. Then select any neighbor $p^{\prime}$ of $v_{1}$ if $v_{1}$ has degree greater than one. Let $v_{2} \in B$ be a neighbor of $p^{\prime}$, where $v_{2} \neq v_{1}$ if such a neighbor exists. Otherwise, let $v_{2} \in B$ be any vertex other than $v_{1}$. If $p$ has degree two, then let $v_{1}, v_{2} \in B$ be the two neighbors of $p$.

Construct a new graph $F\left(A^{\prime}, B^{\prime}\right)$ by deleting $p$ and its incident edge from $F(A, B)$. Replace $v_{1}$ and $v_{2}$ by a single vertex $v$, where $v$ is adjacent to all vertices that were adjacent to either $v_{1}$ or $v_{2}$. The vertex weights in this new graph are

$$
u_{j}^{\prime}= \begin{cases}u_{v_{1}}+u_{v_{2}}, & j=v \\ u_{j}, & j \in B \backslash\left\{v_{1}, v_{2}\right\} .\end{cases}
$$

With this specification, $U^{\prime}=\sum_{j \in B^{\prime}} u_{j}^{\prime}=U$. Thus, $A^{\prime}=A \backslash\{p\}$ and $B^{\prime}=B \cup\{v\} \backslash\left\{v_{1}, v_{2}\right\}$. By assumption, there is a $K-1$ cover, $S^{\prime}$ such that

$$
\begin{aligned}
(2 b-K-a) \sum_{j \in B^{\prime} \cap S} u_{j}^{\prime} & =\left(2\left|B^{\prime}\right|-(K-1)-\left|A^{\prime}\right|\right) \sum_{j \in B^{\prime} \cap S} u_{j}^{\prime} \\
& \geq\left(K-1-\left|A^{\prime}\right|\right) U \\
& =(K-a) U .
\end{aligned}
$$

If $v \notin S^{\prime}$, then $S=S^{\prime} \cup\{p\}$ is a $K$-cover for $F(A, B)$, where $\sum_{j \in B \cap S} u_{j}=\sum_{j \in B^{\prime} \cap S^{\prime}} u_{j}^{\prime}$. If 
$v \in S^{\prime}$, then $S=S^{\prime} \cup\left\{v_{1}, v_{2}\right\} \backslash\{v\}$ is a $K$ cover for $F(A, B)$ with $\sum_{j \in B \cap S} u_{j}=\sum_{j \in B^{\prime} \cap S^{\prime}} u_{j}^{\prime}$. In either case, $F(A, B)$ satisfies the conditions of the lemma.

Consequently, we suppose that all vertices of $A$ in $F(A, B)$ have degree at least three. From Lemma 1, there is a $K$ cover $S$ such that $|S \cap B| \geq 2 K-b$. Since $F(A, B)$ is a counterexample to the lemma,

$$
(2 b-K-a) \sum_{j \in B \backslash S} u_{j}>(2 b-K-a) U-(K-a) U=2(b-K) U .
$$

Let $t=\operatorname{argmax}_{i \in B \backslash S}\left\{u_{i}\right\}$. Because $|B \backslash S| \leq 2(b-K)$ and $(2 b-K-a) \sum_{j \in B \backslash S} u_{j}>$ $2(b-K) U,(2 b-K-a) u_{t}>U$. Construct a new graph $F(A, B \backslash\{t\})$ by deleting $t$ and its incident edges. Since the induction hypothesis applies to $F(A, B \backslash\{t\})$ and $a<K<b$, there is a $K-1$ cover $S^{\prime}$ where $(2 b-K-a-1) \sum_{j \in B \cap S^{\prime}} u_{j}^{\prime} \geq U^{\prime}(K-a-1)$.

Observe that $S=S^{\prime} \cup\{t\}$ is a $K$-cover for $F(A, B)$. Also,

$$
\begin{aligned}
(2 b-K-a) \sum_{j \in B \cap S} u_{j} & =(2 b-K-a)\left(\sum_{j \in(B \cap S) \backslash\{t\}} u_{j}^{\prime}+u_{t}\right) \\
& \geq(2 b-K-a)\left(\frac{K-a-1}{2 b-K-a-1} U^{\prime}+u_{t}\right) \\
& =(2 b-K-a)\left(\frac{K-a-1}{2 b-K-a-1}\left(U-u_{t}\right)+u_{t}\right) \\
& \geq \frac{(2 b-K-a)(K-a-1) U+(2 b-2 K)(2 b-K-a) u_{t}}{2 b-K-a-1} \\
& >\frac{(2 b-K-a)(K-a-1) U+(2 b-2 K) U}{2 b-K-a-1} \\
& =(K-a) U .
\end{aligned}
$$

Thus, $F(A, B)$ satisfies the lemma. Contradiction.

For the original graph, consider the solution $x^{A}$ associated with the set of medians $A$. For each $j \in A$, let $N_{j}\left(x^{A}\right)$ be the set of vertices assigned to $j$. Similarly, define $x^{B}$ and $N_{j}\left(x^{B}\right)$ for each $j \in B$. Construct a bipartite graph $H(A, B)$ with $a+b$ vertices. The edge 
$(i, j)$ exists if $N_{i}\left(x^{A}\right) \cap N_{j}\left(x^{B}\right) \neq \emptyset$. Notice that $H(A, B)$ does not have any isolated vertices or any cycles. A cycle in $H(A, B)$ implies a cycle in the underlying tree. Thus, $H(A, B)$ is acyclic.

To apply Lemma 2 to $H(A, B)$, we assign weights to the vertices of $B$. Let

$$
\begin{aligned}
\phi_{A}(i) & =\text { the distance of } i \text { to nearest median in } A \\
\phi_{B}(i) & =\text { the distance of } i \text { to nearest median in } B \\
u_{j} & = \begin{cases}\sum_{i \in A} \sum_{k \in N_{i}\left(x^{A}\right) \cap N_{j}\left(x^{B}\right)}\left\{\phi_{A}(k)-\phi_{B}(k)\right\}, & j \in B \backslash A \\
0, & \text { otherwise. }\end{cases}
\end{aligned}
$$

For $j \in B \backslash A, u_{j}$ is the distance savings for assigning all vertices of $N_{j}\left(x^{B}\right)$ to $j$ instead of the medians of $A$. Note that $u_{j}$ might be negative and that $U=\sum_{j \in B} u_{j}=z(A)-z(B)$.

Using the cover established by Lemma 2 as the set of medians, we construct a solution to (IP). If edge $(i, j)$ is covered by $j \in B$, then assign the vertices in $N_{i}\left(x^{A}\right) \cap N_{j}\left(x^{B}\right)$ to $j$. If $(i, j)$ is covered by $i \in A \backslash B$, then assign the vertices $N_{i}\left(x^{A}\right) \cap N_{j}\left(x^{B}\right)$ to $i$.

Theorem $9 z^{*} / z^{L P}<2-2 /(K+1)$.

Proof. Suppose we are given a vertex cover $S$ that satisfies Lemma 2. From the definition of $u_{j}$,

$$
z(S)=z(A)-\sum_{j \in B \cap S} u_{j}
$$

From Lemma 2,

$$
z(S) \leq z(A)-\frac{K-a}{2 b-K-a}[z(A)-z(B)]=\frac{2(b-K) z(A)+(K-a) z(B)}{2 b-K-a} .
$$

Since $\lambda=(b-K) /(b-a)$,

$$
\begin{aligned}
z^{L P} & =\lambda z(A)+(1-\lambda) z(B) \\
& =\frac{b-K}{b-a} z(A)+\left(1-\frac{b-K}{b-a}\right) z(B) \\
& =\frac{(b-K) z(A)+(K-a) z(B)}{b-a} .
\end{aligned}
$$


Hence,

$$
\begin{aligned}
\frac{z^{*}}{z^{L P}} & \leq \frac{z(S)}{z^{L P}} \\
& \leq \frac{[2(b-K) z(A)+(K-a) z(B)] /(2 b-K-a)}{[(b-K) z(A)+(K-a) z(B)] /(b-a)} \\
& <\frac{2(b-a)}{2 b-K-a} \\
& \leq \frac{2((K+1)-1)}{2(K+1)-K-1} \\
& =2-\frac{2}{K+1}
\end{aligned}
$$

The last inequality follows because the fraction is maximized when $b=K+1$ and $a=1$.

For the problem where only a subset of vertices are assigned to medians, [8] presents an example which shows that (7) is asymptotically tight. For our problem, a slightly different example is needed. For a specified $K$, we construct a $v$-star, where $v \geq 2, c$ has degree $K+1$, each path from $c$ to a leaf node has $v$ nodes, each arc incident to $c$ has a distance of 1 , and all other arcs have distance $\epsilon=2 /(v+1) v(v-1)$. The optimal integer solution is to assign medians to $c$ and to $K-1$ of the vertices adjacent to $c$. The distance of this solution is $2 v+1$. The optimal LP solution assigns $1 / K$ to $c$ and $(K-1) / K$ to each of the $K+1$ medians adjacent to $c$. The value of the LP solution is $(K+1) v / K+(K-1) / K+1$. Thus,

$$
\begin{aligned}
\frac{z^{*}}{z^{L P}} & =\frac{2 v+1}{(K+1) v / K+(K-1) / K+1} \\
& =2-\frac{2 v+3 K-2}{v(K+1)+K-1} .
\end{aligned}
$$

As $v$ goes to $\infty$, the right hand side of $(8)$ goes to $2-2 /(K+1)$.

\section{Restricting the Feasible Region}

Theorem 1 establishes that $Q_{K}$ is not integral when $n \geq 4$. In this section, we add a class of constraints that restricts $Q_{K}$, while retaining an optimal solution. Then, we find the types 
of trees for which this new polytope is integral.

Theorem 10 For a general graph, there exists an optimal solution $x^{*}$ to either (IP) or (LP) where $x_{i l}^{*}=0$ for all $l$ such that $l$ is a leaf node and $i \neq l$.

Proof. From the path consistency constraints, if $x_{i l}^{*}>0$ for some leaf node $l$, then $x_{q l}^{*}>0$ where $q$ is the unique neighbor of $l$. Consider the solution $x^{\prime}$ where

$$
x_{j j}^{\prime}= \begin{cases}x_{q q}^{*}+x_{q l}^{*}, & j=q \\ x_{l l}^{*}-x_{q l}^{*}, & j=l \\ x_{j j}^{*}, & j \neq q, l,\end{cases}
$$

and all other assignments are made according to (1). This solution is feasible and has a value no larger than the value of $x^{*}$.

Theorem 10 implies that we can add the following constraints

$$
x_{i l}=0 \quad \text { for all } i \in V \text { and leaf nodes } l \text { where } i \neq l
$$

to the K-median formulation.

Let $\hat{P}(V)=\hat{P}=P \cap\{x: x$ satisfies (9) $\}$. Also, let $\hat{Q}_{K}=\hat{P} \cap\left\{x: \sum_{j \in V} x_{j j}=K\right\}$.

Remark If $Q_{K}$ is integral, then $\hat{Q}_{K}$ is integral. Moreover, the converse is not true.

Properties 1 and 2 can be extended in a straightforward way to $\hat{Q}$ and $\hat{P}$. We define these new results as Property $1^{\prime}$ and Property $2^{\prime}$, respectively.

The next result establishes when $\hat{Q}_{K}$ is an integral polytope.

Theorem $11 \hat{Q}_{K}$ is integral for all $K$ iff $G$ is a 1-star.

Proof. $\Rightarrow$. If $n=3$, then $G$ is a 1-star. If $n \geq 4$ and $G$ is not a 1-star, then $G$ has an embedded path of at least four vertices. The example of a path presented in Theorem 1 shows that $\hat{Q}_{K}$ is not integral. 
$\Leftarrow$. Let $x^{0}$ be an extreme point solution to (LP). Because all vertices in $V \backslash\{c\}$ are leaf nodes, constraints (9) establish that $x_{c j}^{0}=0$ for $j \in V \backslash\{c\}$. Thus, $x_{c c}^{0}=1$. By Property $2^{\prime}$, there is at most one leaf with a fractional median. Since $K$ is integral, this is not possible.

Note that the polytope is not integral if constraints (9) are not included in the formulation (see Theorem 1).

\section{Facets for General Graphs}

In this section, we introduce a class of facets for the $K$-median problem on a general graph. They are not contained in the class identified by Avella and Sassano [2] nor are they subsumed by the facets identified for the uncapacitated facility location problem (see for example Cornuejols and Thizy [5] or Cho et al. [4]). The facets we identify in the next result are used in the next section to give a complete polyhedral description for a class of trees.

Theorem 12 For every $R \subseteq V$ where $K \leq|R|=r$ and for every $S$ where $\emptyset \subseteq S \subseteq V \backslash R$,

$$
\begin{array}{r}
(r-K+1)\left((r-K) \sum_{i \in R} x_{i i}-\sum_{\substack{i, j \in R \\
i \neq j}} x_{i j}\right)+(r-K)\left((r-K+1) \sum_{j \in S} x_{j j}-\sum_{\substack{i \in R \\
j \in S}} x_{i j}\right) \\
\leq(r-K+1)(r-K)(K-1)
\end{array}
$$

is valid.

Proof. Since only integral solutions are considered, $I=M$. First suppose that $M \not \subset R \cup S$. Then, there is at least one median not in $R \cup S$. Therefore,

$$
\sum_{i \in R} x_{i i}+\sum_{i \in S} x_{i i} \leq K-1 .
$$

This implies that

$$
(r-K+1)(r-K)\left(\sum_{i \in R} x_{i i}+\sum_{j \in S} x_{j j}\right) \leq(r-K+1)(r-K)(K-1) .
$$


In the left hand side of (10), only variables $x_{k k}$ for $k \in V$ have positive coefficients. Thus, (10) is established when $M \not \subset R \cup S$.

Alternatively, suppose that $M \subset R \cup S$. Then,

$$
\sum_{i \in R \cup S} x_{i i}=K
$$

Since all medians are contained in $R \cup S$, every vertex in $R \backslash M$ is assigned to a vertex in $R \cup S$. If $S \cap M=\emptyset$, then

$$
\sum_{\substack{i, j \in R \\ i \neq j}} x_{i j}=r-K .
$$

Thus, an upper bound on the left hand side of (10) is

$$
(r-K+1)(r-K) K-(r-K+1)(r-K)=(r-K+1)(r-K)(K-1) .
$$

If $S \cap M \neq \emptyset$, then

$$
\begin{aligned}
(r-K+1) \sum_{\substack{i, j \in R \\
i \neq j}} x_{i j}+(r-K) \sum_{\substack{i \in R \\
j \in S}} x_{i j} & \geq(r-K) \sum_{i \in R} \sum_{\substack{j \in S \cup R \\
i \neq j}} x_{i j} \\
& \geq(r-K)(r-K+1) .
\end{aligned}
$$

An upper bound for the left hand side of (10) is

$$
(r-K)(r-K+1) K-(r-K)(r-K+1)=(r-K)(r-K+1)(K-1) .
$$

We now show that an inequality (10) for $K<r$ and $\emptyset \subset S \subset V \backslash R$ generates a facet $\mathcal{F}$ of the K-median polytope. For a given inequality, we consider three types of median assignments that define points in $\mathcal{F}$ :

Type 1: All $K$ medians are in $R$. The left hand side of inequality $(10)$ is $(r-K+1)((r-$ $K) K-(r-K))=(r-K)(r-K+1)(K-1)$. 
Type 2: $K-1$ medians are in $R \cup S$. All non-median vertices in $R$ are assigned to the median in $\overline{(R \cup S)}$. The left hand side of inequality $(10)$ is $(r-K+1)(r-K) \sum_{i \in R \cup S} x_{i i}=$ $(r-K+1)(r-K)(K-1)$.

Type 3: $K-1$ medians are in $R$, the last median is in $S$, and all non-median vertices in $R$ are assigned to the median in $S$. The left hand side of inequality (10) is $(r-K+$ 1) $(r-K)(K-1)+(r-K)[(r-K+1)-(r-K+1)]=(r-K+1)(r-K)(K-1)$.

Since these points satisfy (10) at equality, $\mathcal{F}$ generates a face of the integer polytope of the constraint set of (IP).

To simplify notation, assume that $R=\{1,2, \ldots, r\}$ and $S=\{n-|S|+1, n-|S|+2, \ldots, n\}$, where $r+1 \notin S$.

For a given $\mathcal{F}$ defined by (10), let $\pi^{\prime} x \leq \pi_{0}^{\prime}$ be valid for the polytope and generate a face that contains $\mathcal{F}$. Construct

$$
\pi x \leq \pi_{0},
$$

which induces the same face as $\pi^{\prime} x \leq \pi_{0}^{\prime}$, by adding multiples of $\sum_{j=1}^{n} x_{i j}=1$ for $i \in R$ so that $\pi_{i, r+1}=0$. Then for $i \in \bar{R}$, add multiples of $\sum_{j=1}^{n} x_{i j}=1$ so that $\pi_{i 1}=0$. Also, add a multiple of $\sum_{i=1}^{n} x_{i i}=K$ so that $\pi_{r+1, r+1}=0$. Finally, multiply the resulting inequality with a positive number so that

$$
\pi_{r r}=\delta(r-K)(r-K+1)
$$

where $\delta \in\{-1,0,1\}$. (Later we show that $\delta \in\{0,1\}$.)

To determine the possible values of $\pi$ and $\pi_{0}$, three kinds of arguments are used:

Argument 1 Suppose $x$ is a feasible integer solution where $x_{i j}=x_{l l}=1$ for $i \neq j \neq l$. Let $x_{i j}^{\prime}=0, x_{i l}^{\prime}=1$, and $x_{k q}^{\prime}=x_{k q}$ for all $(k, q) \neq(i, j)$ or $(i, l)$. If $x, x^{\prime} \in \mathcal{F}$, then $\pi_{i j}=\pi_{i l}$.

Proof. Since $x, x^{\prime} \in \mathcal{F}$, they satisfy (11) with equality. Therefore, $\pi x=\pi x^{\prime}$ and $\pi_{i j} x_{i j}=$ $\pi_{i l} x_{i l}^{\prime}$. 
Argument 2 Suppose $x$ is a feasible integer solution where $x_{i j}=x_{l l}=1$ for $j \neq i, l$, and where $x_{h l}=0$ for all $h \neq l$. Let $x_{i j}^{\prime}=x_{l l}^{\prime}=0, x_{l j}^{\prime}=x_{i i}^{\prime}=1$, and $x_{k q}^{\prime}=x_{k q}$ for all $(k, q) \neq(i, i),(i, j),(l, l)$, or $(l, j)$. If $x, x^{\prime} \in \mathcal{F}$, then $\pi_{i j}+\pi_{l l}=\pi_{i i}+\pi_{l j}$.

Proof. Since $x, x^{\prime} \in \mathcal{F}$, they satisfy (11) with equality. Therefore, $\pi x=\pi x^{\prime}$ and $\pi_{i j} x_{i j}+$ $\pi_{l l} x_{l l}=\pi_{i i} x_{i i}^{\prime}+\pi_{l j} x_{l j}^{\prime}$

Argument 3 Suppose $x$ is a feasible integer solution where $x_{i j}=x_{j j}=1$ for $j \neq i$, and where $x_{l j}=0$ for all $l \neq i, j$. Let $x_{i j}^{\prime}=x_{j j}^{\prime}=0, x_{j i}^{\prime}=x_{i i}^{\prime}=1$, and $x_{k q}^{\prime}=x_{k q}$ for all $(k, q) \neq(i, i),(i, j),(j, j)$, or $(j, i)$. If $x, x^{\prime} \in \mathcal{F}$, then $\pi_{i j}+\pi_{j j}=\pi_{i i}+\pi_{j i}$.

Proof. Since $x, x^{\prime} \in \mathcal{F}$, they satisfy (11) with equality. Therefore, $\pi x=\pi x^{\prime}$ and $\pi_{i j} x_{i j}+$ $\pi_{j j} x_{j j}=\pi_{i i} x_{i i}^{\prime}+\pi_{j i} x_{j i}^{\prime}$.

Lemma $3 \pi_{i j}=\pi_{i l}$ for $i \in V$ and $j, l \in R \backslash\{i\}$.

Proof. Consider the incidence vector $x$ with the following Type 1 assignment: medians are at $j, l$ and at $K-2$ additional vertices in $R \backslash\{i\}$, vertex $i$ is assigned to $j$, and all other vertices are assigned to arbitrary medians.

Let the assignment $x^{\prime}$ only differ from $x$ in that vertex $i$ is assigned to median $l$. Since $x^{\prime}$ has a Type 1 assignment, $x^{\prime} \in \mathcal{F}$. Use Argument 1 for vertex $i$ and medians $j$ and $l$ to conclude that $\pi_{i j}=\pi_{i l}$.

Lemma $4 \pi_{i j}=0$, for $i \in \bar{R}$ and $j \in V \backslash\{i\}$.

Proof. We first establish that $\pi_{i j}=\pi_{i l}$ for $i \in \bar{R}, j \in R$, and $l \in \bar{R} \backslash\{i\}$. There are two cases: $l \in S \backslash\{i\}$ and $l \in \overline{(R \cup S)} \backslash\{i\}$.

Case 1. $l \in S \backslash\{i\}$.

Consider the incidence vector $x$ with the following Type 3 assignment: medians are at $j, l$ and at $K-2$ additional vertices in $R$, vertex $i$ is assigned to $j$, and all other vertices are assigned to $l$. 
Let the assignment $x^{\prime}$ only differ from $x$ in that vertex $i$ is assigned to median $l$. Since $x^{\prime}$ has a Type 3 assignment, $x^{\prime} \in \mathcal{F}$. Use Argument 1 for vertex $i$ and medians $j$ and $l$ to conclude that $\pi_{i j}=\pi_{i l}$.

Case 2. $l \in \overline{(R \cup S)} \backslash\{i\}$.

Consider the incidence vector $x$ with the following Type 2 assignment: medians are at $j, l$ and at $K-2$ additional vertices in $R$, vertex $i$ is assigned to $j$, and all other vertices are assigned to $l$.

Let the assignment $x^{\prime}$ only differ from $x$ in that vertex $i$ is assigned to median $l$. Since $x^{\prime}$ has a Type 2 assignment, $x^{\prime} \in \mathcal{F}$. Use Argument 1 for vertex $i$ and medians $j$ and $l$ to conclude that $\pi_{i j}=\pi_{i l}$.

Thus, $\pi_{i j}=\pi_{i l}$ for $i \in \bar{R}, j \in R$, and $l \in \bar{R} \backslash\{i\}$. Using Lemma $3, \pi_{i j}=\pi_{i l}$ for $i \in \bar{R}$ and $j, l \neq i$. By construction, $\pi_{i 1}=0$ for $i \in \bar{R}$. Hence, $\pi_{i j}=0$, for $i \in \bar{R}$, and $j \in V \backslash\{i\}$.

Lemma $5 \pi_{i j}=-\delta(r-K+1)$ for $i, j \in R$ and $i \neq j$.

Proof. First, we show that $\pi_{i l}=\pi_{j l}$, for $i, j \in R$ and $l \in R \backslash\{i, j\}$.

Let $J \subset R$ and $l \in R \backslash J$ where $|J|=r-K$. Consider the incidence vector $x$ with the following Type 2 assignment: a median is at $r+1$, and at the $K-1$ vertices in $R \backslash(J \cup\{l\})$, vertices in $J \cup\{l\}$ are assigned to $r+1$, and all other vertices are assigned to arbitrary medians other than $r+1$.

Let the assignment $x^{\prime}$ only differ from $x$ in that there is a median at $l$ instead of at $r+1$, and the vertices in $J$ are assigned to $l$. Since $x^{\prime}$ has a Type 1 assignment, $x^{\prime} \in \mathcal{F}$. From the construction of $\pi, \sum_{i \in J \cup\{l\} \cup\{r+1\}} \pi_{i, r+1}=0$. From Lemma $4, \pi_{r+1, l}=0$. Thus, $\pi x^{\prime}=\pi x$ implies that $\sum_{i \in J \cup\{l\}} \pi_{i l}=0$.

Now, consider two subsets $J, J^{\prime} \subseteq R$ of cardinality $r-K$, where $J \backslash J^{\prime}=\{i\}$ and $J^{\prime} \backslash J=$ $\{j\}$. We have that $\sum_{k \in J \cup\{l\}} \pi_{k l}=0=\sum_{k \in J^{\prime} \cup\{l\}} \pi_{k l}$. Since $\sum_{k \in J \cup\{l\}} \pi_{k l}-\sum_{k \in J^{\prime} \cup\{l\}} \pi_{k l}=$ $\pi_{i l}-\pi_{j l}, \pi_{i l}=\pi_{j l}$ 
We now establish that $\pi_{i r}=-\delta(r-K+1)$ for $i \in R \backslash\{r\}$. The above analysis implies that $\sum_{j \in J \cup\{r\}} \pi_{j r}=(r-K) \pi_{i r}+\pi_{r r}$, where $i \in J, r \notin J$ and $l=r$. From the construction of $\pi, \pi_{r r}=\delta(r-K)(r-K+1)$. Because $\sum_{j \in J \cup\{r\}} \pi_{j r}=0$, we have that $(r-K) \pi_{i r}+\delta(r-$ $K)(r-K+1)=0$. Now, $r-K>0$ implies that $\pi_{i r}=-\delta(r-K+1)$.

From Lemma $3, \pi_{i j}=-\delta(r-K+1)$ for $i \in R \backslash\{r\}, j \in R \backslash\{r\}$ and $i \neq j$. With the previous observation $\pi_{i l}=\pi_{j l}$ applied to $\pi_{r l}=\pi_{j l}$ establishes that $\pi_{r j}=-\delta(r-K+1)$ for $j \in R \backslash\{r\}$.

Lemma $6 \pi_{i i}=\delta(r-K)(r-K+1)$ for $i \in R \cup S$.

Proof. For $i=r$, the lemma is true by construction of $\pi$. For $i \neq r$, there are two cases: $i \in R \backslash\{r\}$ and $i \in S$.

Case 1. $i \in R \backslash\{r\}$.

Consider the incidence vector $x$ with the following Type 1 assignment: medians are at $r$, and at $K-1$ additional vertices in $R \backslash\{i\}$, vertex $i$ is assigned to $r$, and all other vertices are assigned to arbitrary medians other than $r$.

Let the assignment $x^{\prime}$ only differ from $x$ in that there is a median at vertex $i$ instead of at $r$, and $r$ is assigned to $i$. Since $x^{\prime}$ has a Type 1 assignment, $x^{\prime} \in \mathcal{F}$. Use Argument 3 to conclude that $\pi_{r r}+\pi_{i r}=\pi_{r i}+\pi_{i i}$. By the construction of $\pi, \pi_{r r}=\delta(r-K)(r-K+1)$, and by Lemma $5, \pi_{i r}=-\delta(r-K+1)=\pi_{r i}$. Hence, $\pi_{i i}=\delta(r-K)(r-K+1)$.

Case 2: $i \in S$.

Consider the incidence vector $x$ with the following Type 2 assignment: medians are at $r$, $r+1$, and at $K-2$ additional vertices in $R$, all other vertices are assigned to $r+1$.

Let the assignment $x^{\prime}$ only differ from $x$ in that there is a median at $i$ instead of at $r$, and $r$ is assigned to $r+1$. Since $x^{\prime}$ has a Type 2 assignment, $x^{\prime} \in \mathcal{F}$. Use Argument 2 to conclude that $\pi_{i, r+1}+\pi_{r r}=\pi_{i i}+\pi_{r, r+1}$. By the construction of $\pi, \pi_{r r}=\delta(r-K)(r-K+1)$ and $\pi_{r, r+1}=0$. From Lemma $4, \pi_{i, r+1}=0$. Hence, $\pi_{i i}=\delta(r-K)(r-K+1)$. 
Lemma $7 \pi_{i l}=0$ for $i \in R$ and $l \in \overline{(R \cup S)}$.

Proof. Consider the incidence vector $x$ with the following Type 2 assignment: medians are at $i \in R, l \in \overline{(R \cup S)}$, and at $K-2$ additional vertices in $R \backslash\{i\}$, all non-median vertices are assigned to $l$.

Let the assignment $x^{\prime}$ only differ from $x$ in that there is a median at $n \in S$ instead of at $i$, and $i$ is assigned to $l$. Since $x^{\prime}$ has a Type 2 assignment, $x^{\prime} \in \mathcal{F}$. Use Argument 2 to conclude that $\pi_{n l}+\pi_{i i}=\pi_{n n}+\pi_{i l}$. From Lemma 6, $\pi_{i i}=\pi_{n n}=\delta(r-K)(r-K+1)$. Thus, $\pi_{i l}=\pi_{n l}$. Since Lemma 4 implies that $\pi_{n l}=0, \pi_{i l}=0$.

Lemma $8 \pi_{l l}=0$ for $l \in \overline{(R \cup S)}$.

Proof. Consider the incidence vector $x$ with the following Type 2 assignment: medians are at $r+1$, and at $K-1$ additional vertices in $R$, all non-median vertices are assigned to $r+1$.

Let the assignment $x^{\prime}$ only differ in that there is a median at $l \in \overline{(R \cup S)}$ instead of at $r+1$, and all non-median vertices are assigned to $l$. Since $x^{\prime}$ is a Type 2 assignment, $x^{\prime} \in \mathcal{F}$. From Lemmas 4 and $7, \pi_{i j}=0$ for $j \in\{r+1, l\}$ and $i \in V \backslash\{j\}$. Thus, $\pi x=\pi x^{\prime}$ implies that $\pi_{r+1, r+1} x_{r+1, r+1}=\pi_{l l} x_{l l}^{\prime}$. Since $\pi_{r+1, r+1}=0$ and $x_{l l}=1$, we conclude that $\pi_{l l}=0$.

Lemma $9 \pi_{i l}=-\delta(r-K)$ for $i \in R$ and $l \in S$.

Proof. First, we show that $\pi_{i l}=\pi_{j l}$ for $i, j \in R$ and $l \in S$. Consider the incidence vector $x$ with the following Type 3 assignment: medians are at $j \in R, l \in S$ and at $K-2$ additional vertices in $R \backslash\{i\}$, all non-median vertices are assigned to $l$.

Let the assignment $x^{\prime}$ only differ from $x$ in that there is a median at vertex $i$ instead of at $j$, and $j$ is assigned to median $l$. Since $x^{\prime}$ is a Type 3 assignment, $x^{\prime} \in \mathcal{F}$. Use Argument 2 to conclude that $\pi_{i l}+\pi_{j j}=\pi_{i i}+\pi_{j l}$. From Lemma $6, \pi_{i i}=\delta(r-K)(r-K+1)=\pi_{j j}$. Hence, $\pi_{i l}=\pi_{j l}$ for $i, j \in R$ and $l \in S$. 
Now, consider the incidence vector $x$ with the following Type 1 assignment: medians are at $i \in R$ and at $K-1$ additional vertices in $R \backslash\{i\}$, all non-median vertices are assigned to $i$.

Let the assignment $x^{\prime}$ only differ from $x$ in that there is a median at vertex $l \in S$ instead of at $i$, and all non-median vertices are assigned to $l$. Since $x^{\prime}$ is a Type 3 assignment, $x^{\prime} \in \mathcal{F}$. Consequently, $\pi x=\pi x^{\prime}$. Thus,

$$
\sum_{j \in R \backslash M} \pi_{j i}+\pi_{i i}+\sum_{j \in \bar{R}} \pi_{j i}=\sum_{j \in R \backslash M} \pi_{j l}+\pi_{i l}+\sum_{j \in \bar{R} \backslash\{l\}} \pi_{j l}+\pi_{l l} .
$$

Now, $\pi_{j i}=\pi_{j l}=0$ for $j \in \bar{R}$, from Lemma 4, and $\pi_{i i}=\pi_{l l}$ from Lemma 6. As a result,

$$
\sum_{j \in R \backslash M} \pi_{j i}=\sum_{j \in R \backslash M} \pi_{j l}+\pi_{i l} .
$$

Because $|R \backslash M|=r-K, \pi_{j i}=-\delta(r-K+1)$ for $j \in R \backslash M$ from Lemma 5 , and $\pi_{j l}=\pi_{i l}$ for $j \in R \backslash M$, we have that

$$
\begin{aligned}
-(r-K) \delta(r-K+1) & =(r-K) \pi_{i l}+\pi_{i l} \\
-\delta(r-K) & =\pi_{i l} .
\end{aligned}
$$

We now establish the main result of this section.

Theorem 13 For $K<r$ and $\emptyset \subset S \subset V \backslash R$, the inequalities (10) induce facets for the general $K$-median $(K \geq 2)$ polytope.

Proof. By the construction of $\pi$ and from Lemmas 4-9, the coefficients of inequality (11) are determined up to $\delta$. We first show that $\pi_{0}=\delta(K-1)(r-K)(r-K+1)$.

Consider the incidence vector $x$ with the following Type 2 assignment: medians are at $l \in \overline{(R \cup S)}$, and at $K-1$ vertices in $R$, all non-median vertices are assigned to $l$. Then,

$$
\begin{aligned}
\pi_{0} & =\pi x \\
& =\sum_{i \in R \cap M} \pi_{i i}+\pi_{l l}+\sum_{j \in \bar{R} \backslash M} \pi_{j l}+\sum_{i \in R \backslash M} \pi_{i l} \\
& =(K-1) \delta(r-K)(r-K+1)+0+0+0 .
\end{aligned}
$$


where the last equality follows from Lemmas $6,8,4$, and 7 .

Consider another incidence vector $\hat{x}$ with the following assignment: medians are at $l \in S$, and at $K-1$ vertices in $R$, all non-median vertices are assigned to medians in $R$. While $\hat{x}$ is feasible, this assignment might not be in $\mathcal{F}$. Now,

$$
\begin{aligned}
\pi \hat{x} & =\sum_{i \in(R \cup S) \cap M} \pi_{i i}+\sum_{j \in \bar{R} \backslash M} \sum_{i \in R \cap M} \pi_{j i}+\sum_{j \in R \backslash M} \sum_{i \in R \cap M} \pi_{j i} \\
& =K \delta(r-K)(r-K+1)+0-(r-K+1) \delta(r-K+1) .
\end{aligned}
$$

where the last equality follows from Lemmas 6,4 , and 5 . Because $\pi \hat{x} \leq \pi_{0}=\delta(K-1)(r-$ $K)(r-K+1)$,

$$
\begin{aligned}
K \delta(r-K)(r-K+1)-(r-K+1) \delta(r-K+1) & \leq(K-1) \delta(r-K)(r-K+1) \\
\delta(r-K) & \leq \delta(r-K+1) .
\end{aligned}
$$

Consequently, $\delta \in\{0,1\}$. If $\delta=0$, then $\pi x \leq \pi_{0}$ reduces to $0 x \leq 0$, which is trivially true. Therefore, the original inequality $\pi^{\prime} x \leq \pi_{0}^{\prime}$ is only a linear combination of valid equations for the polytope. As a result, it induces a trivial face (the polytope itself). Alternatively, if $\delta=1$, then the inequality $\pi x \leq \pi_{0}$ is identical to the inequality of type (8) that induces $\mathcal{F}$. Consequently, $\pi x \leq \pi_{0}$ also defines $\mathcal{F}$. Hence, $\mathcal{F}$ is not contained in any other proper face of the polytope and is therefore a facet.

Notice that the condition that $K<r$ is used in Lemma 3 and is needed so that inequality (11) is not trivially true. Also, the condition that $S \neq \emptyset$ is used in Lemma 7.

\section{Stars with at Most Two Vertices Per Branch}

An example of a 2-star is presented in Figure 4. Denote the $K$-median problem on a 2-star as $\left(I P_{2}\right)$ and the associated linear relaxation as $\left(L P_{2}\right)$. We assume that the path consistency constraints and constraints (9) are included in this formulation. In this section, we present 


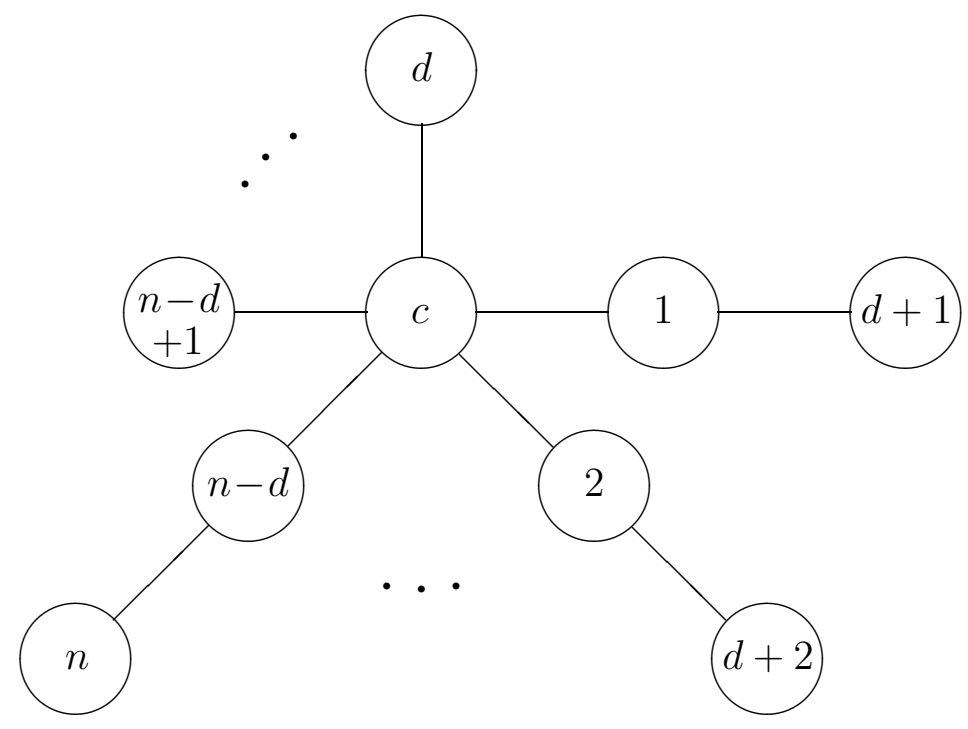

Figure 4: A 2-star.

an integral polyhedral description for $\left(I P_{2}\right)$ that restricts the feasible set, while retaining an optimal solution.

The degree of the center vertex $c$ is $d$, and the set of neighbors of $c$ is $N_{c}=\{1,2, \ldots, d\}$. We label the vertices so that the neighbors of $N_{c} \backslash\{c\}$ are $\{d+1, d+2, \ldots, n\}=L$. Further, if vertex $i \neq c$ is a neighbor of $q \in N_{c}$, then $i=q+d$. A branch is a pair of vertices of the form $(q, q+d)$ where $q \in N_{c}$.

We now establish some properties of an optimal integer solution. From Theorem 5, we restrict the analysis to the cases where $2 \leq K \leq n-1$.

Lemma 10 If $G$ is a 2-star, then $\hat{Q}_{K} \cap\left\{x_{c c}=1\right\}$ is integral.

Proof. From Property 2, an extreme point solution to $\left(L P_{2}\right)$ has at most one branch with fractional medians. However, this branch is a path on three nodes. From Theorem 5, there is an integral assignment of the fractional medians to the branch.

Lemma 11 There exists an optimal integer solution $x^{*}$ to $\left(I P_{2}\right)$ where $x_{i q}^{*}=0$ for $q \in N_{c}$ and for all $i \in V \backslash\{c, q, q+d\}$. 
Proof. Suppose $x_{c c}^{*}=1$. Then, the result follows from path consistency. As a result, we assume that $x_{c c}^{*}=0$. Hence, there is a vertex $q \in V \backslash\{c\}$ such that $x_{c q}^{*}=1$. If we satisfy (9), then $q \in N_{c}$.

The graph induced by the vertices of $N_{q}\left(x^{*}\right) \backslash\{q\}$ forms two subtrees. One subtree has at most one vertex, a leaf node. The other subtree has at least two vertices. Thus, we can set $x_{c c}^{*}=1$ and $x_{q q}^{*}=0$. The value of this new solution is no larger than the original optimal solution and $x_{i q}=0$.

We restrict $\left(I P_{2}\right)$ and $\left(L P_{2}\right)$ to those solutions that satisfy Lemma 11 by adding the equations

$$
x_{i q}=0 \text { for } q \in N_{c} \text { and } i \in V \backslash\{c, q\}
$$

to the formulation. Let $Q_{K}^{\prime}=\hat{Q}_{K} \cap\{x: x$ satisfies $(12)\}$

Remark If $x^{0} \in Q_{K}^{\prime}$ is an extreme point of $\hat{Q}_{K}$, then $x^{0}$ is also an extreme point of $Q_{K}^{\prime}$. Thus, Lemma 10 holds when $\hat{Q}_{K}$ is replaced by $Q_{K}^{\prime}$.

Lemma 12 If $G$ is a 2-star, then $Q_{K}^{\prime} \cap\left\{x_{c c}=0\right\}$ is integral.

Proof. From (9) and Lemma 11, no vertex in one branch is assigned to a median in another branch. As a result, each branch is optimized independently. Since each subproblem is a path on three nodes, Theorem 5 establishes that an extreme point solution is integral.

Suppose an optimal extreme point solution, $x^{*}$, has $x_{c c}^{*}=1$ or 0 . Then by Lemmas 10 or 12, respectively, an optimal extreme point LP solution is integral. Therefore, we consider the case when $x_{c c}^{*}=\theta$ where $0<\theta<1$.

We use (10) to generate a set of valid inequalities. Let

$$
R=N_{c} \cup Y_{t} \quad \text { and } \quad S=L \backslash R,
$$


where $Y_{t} \subset L$ and $\left|Y_{t}\right|=\max \{K-d+t, 0\}$ for $t \in\{1,2, \ldots, n-d\}$. Note that $\sum_{\substack{i \in R \\ j \in S}} x_{i j}=0$ holds from Theorem 10. Substituting $R$ and $S$ into (10) yields

$$
\begin{aligned}
(r-K+1)(r-K)\left(K-x_{c c}\right)-(r-K+1) \sum_{\substack{i, j \in R \\
i \neq j}} x_{i j} & \leq(r-K+1)(r-K)(K-1) \\
(r-K)\left(K-x_{c c}\right)-\sum_{\substack{i, j \in R \\
i \neq j}} x_{i j} & \leq(r-K)(K-1) \\
(r-K)\left(1-x_{c c}\right) & \leq \sum_{\substack{i, j \in R \\
i \neq j}} x_{i j}
\end{aligned}
$$

We now show that adding all equations (10) to $\left(L P_{2}\right)$, where $R$ and $S$ are given in (13), provide a formulation where the polytope is integral.

If $d \geq K+1$, then $Y_{1}=\emptyset$. As a result, $\sum_{\substack{i, j \in R \\ i \neq j}} x_{i j}^{*}=0$. Thus, (14) implies that $x_{c c}^{*} \geq 1$, and $\theta$ cannot be fractional. Consequently, we assume that $d \leq K$. From the definition of $R$, $t=r-K$. Then, inequality (14) is equivalent to

$$
t(1-\theta) \leq \sum_{\substack{i, j \in R \\ i \neq j}} x_{i j}
$$

Because $\left|Y_{t}\right|=r-d, R=N_{c} \cup Y_{t}$, and $x_{i j}=0$ for $i, j \in N_{c}$,

$$
\sum_{\substack{i, j \in R \\ i \neq j}} x_{i j} \leq(r-d)-\sum_{j \in Y_{t}} x_{j j}=(K+t-d)-\sum_{j \in Y_{t}} x_{j j} .
$$

Thus, constraints (14) imply that

$$
\sum_{j \in Y_{t}} x_{j j} \leq K-d+t \theta
$$

where $Y_{t} \subset L$ and $\left|Y_{t}\right|=K-d+t$. When inequalities (15) are included in the formulation for $\left(L P_{2}\right)$, Property 1 and Property 2 may no longer hold. This can happen only if an inequality (15) is tight at an optimal solution $x^{*}$.

The next result provides a lower bound for $x_{q q}^{*}$.

Lemma 13 For $q \in N_{c}, x_{q q}^{*} \geq 1-\theta$. 
Proof. From (12), $x_{q i}^{*}=0$ for $q \in N_{c}$ and $i \in V \backslash\{c\}$. Since $x_{c c}^{*}=\theta$ and $\sum_{i \in V} x_{q i}^{*}=1$, the result follows.

Given an extreme point solution $x^{*}$, Lemma 13 implies that the branches of the 2 -star can be partitioned into the following sets:

$$
\begin{aligned}
& A^{1}=\left\{q \in N_{c}: 1-\theta<x_{q q}^{*}<1\right\} \\
& A^{2}=\left\{q \in N_{c}: 0<x_{q+d, q+d}^{*}<\theta, x_{q q}^{*} \in\{1-\theta, 1\}\right\} \\
& A^{3}=\left\{q \in N_{c}: x_{q q}^{*}=1-\theta, \theta<x_{q+d, q+d}^{*}<1\right\} \\
& A^{4}=\left\{q \in N_{c}: x_{q q}^{*}=1, \theta<x_{q+d, q+d}^{*}<1\right\} \\
& A^{5}=\left\{q \in N_{c}: x_{q q}^{*}=1-\theta, x_{q+d, q+d}^{*}=0\right\} \\
& A^{6}=\left\{q \in N_{c}: x_{q q}^{*}=1-\theta, x_{q+d, q+d}^{*}=\theta\right\} \\
& A^{7}=\left\{q \in N_{c}: x_{q q}^{*}=1-\theta, x_{q+d, q+d}^{*}=1\right\} \\
& A^{8}=\left\{q \in N_{c}: x_{q q}^{*}=1, x_{q+d, q+d}^{*}=0\right\} \\
& A^{9}=\left\{q \in N_{c}: x_{q q}^{*}=1, x_{q+d, q+d}^{*}=\theta\right\} \\
& A^{10}=\left\{q \in N_{c}: x_{q q}^{*}=1, x_{q+d, q+d}^{*}=1\right\} .
\end{aligned}
$$

We first establish some properties of these sets and show that some of them are empty.

In what follows, we exploit the argument of transferring $\epsilon$ between medians.

Lemma 14 If $q+d \in Y_{t}$ for $t \in\{1,2, \ldots, n-d\}$ and $x_{q+d, q+d}^{*} \notin\{\theta, 1\}$, then the constraint (15) associated with $Y_{t}$ is loose. 
Proof. Suppose that $x_{q+d, q+d}^{*}<\theta$. If $\sum_{j \in Y_{t}} x_{j j}^{*}=K-d+t \theta$ where $t \in\{2,3, \ldots, n-d\}$, then $\sum_{j \in Y_{t} \backslash\{q+d\}} x_{j j}^{*}>K-d+(t-1) \theta$. Feasibility is violated for constraint (15) associated with the set $Y_{t} \backslash\{q+d\}$. When $q+d \in Y_{1}$, it is not possible that $\sum_{j \in Y_{1}} x_{j j}^{*}=K-d+\theta$. Hence, if $x_{q+d, q+d}<\theta$, then $q+d \notin Y_{t}$.

Suppose that $\left|\left\{l \in Y_{t}: \theta<x_{l l}^{*}<1\right\}\right|=1$. Then, all other elements of $Y_{t}$ have values $\theta$ or 1 . Since $\left|Y_{t}\right|=K-d+t$ and $t(1-\theta) \leq \sum_{i, j \in R, i \neq j} x_{i j}$, we have that $\left|\left\{l \in Y_{t}: x_{l l}^{*}=1\right\}\right| \leq K-d$. As a result, it is not possible that $\sum_{j \in Y_{t}} x_{j j}^{*}=K-d+t \theta$. Therefore, we assume that $\left|\left\{l \in Y_{t}: \theta<x_{l l}^{*}<1\right\}\right| \geq 2$.

Let $\bar{t}=\max \left\{i\right.$ : constraint (15) is tight for $\left.Y_{i}\right\}$. If $l \in L \backslash Y_{\bar{t}}$ and $x_{l l}^{*} \geq \theta$, then $Y_{\bar{t}} \cup\{l\}$ is tight for (15). Because this contradicts the definition of $\bar{t}$, we assume that $x_{l l}^{*}<\theta$ for all $l \in L \backslash Y_{\bar{t}}$. Thus, if $Y_{i}$ is the associated set for any tight constraint (15), then $Y_{i} \subseteq Y_{\bar{t}}$. Also, $l \in Y_{\bar{t}} \backslash Y_{i}$ implies that $x_{l l}^{*}=\theta$.

Let $l_{1}, l_{2} \in\left\{l \in Y_{t}: \theta<x_{l l}^{*}<1\right\}$. For a suitably small $\epsilon>0$, construct a new solution $x^{1}$ from $x^{*}$ by transferring $\epsilon$ from median $l_{1}$ to median $l_{2}$, i.e.

$$
\begin{aligned}
& x_{l_{1} l_{1}}^{1}=x_{l_{1} l_{1}}^{*}-\epsilon \\
& x_{l_{2} l_{2}}^{1}=x_{l_{2} l_{2}}^{*}+\epsilon \\
& x_{l_{1}, l_{1}-d}^{1}=x_{l_{1}, l_{1}-d}^{*}+\epsilon \\
& x_{l_{2}, l_{2}-d}^{1}=x_{l_{2}, l_{2}-d}^{*}-\epsilon \\
& x_{i j}^{1}=x_{i j}^{*} \text { for all other variables. }
\end{aligned}
$$

Notice that no component of $x^{*}$ is driven to zero by this change. Further, no component of $x^{*}$ that is zero becomes non-zero. Also, $x^{1}$ satisfies all the same constraints that $x^{*}$ does.

Now, consider an alternative solution $x^{2}$ where $-\epsilon$ is transferred from median $l_{1}$ to median $l_{2}$ in $x^{*}$. Again, $x^{2}$ is a feasible solution. However, $x^{*}$ is a convex combination of $x^{1}$ and $x^{2}$. Thus, $x^{*}$ is not an extreme point solution. Contradiction.

Lemma 15 For $q \in A^{1}, x_{q+d, q+d}^{*} \in\{0, \theta, 1\}$. 
Proof. For a suitably small $\epsilon>0$, construct a new solution $x^{1}$ from $x^{*}$ by transferring $\epsilon$ from median $q$ to median $q+d$. No component of $x^{*}$ is driven to zero by this change. Further, no component of $x^{*}$ that is zero becomes non-zero. If $x_{q+d, q+d}^{*} \notin\{\theta, 1\}$, then Lemma 14 implies that the associated constraint (15) is loose. Thus, $x^{1}$ satisfies all the same constraints that $x^{*}$ does.

Consider an alternative solution $x^{2}$ where $-\epsilon$ is transferred from median $q$ to median $q+d$ in $x^{*}$. Again, $x^{2}$ is a feasible solution. However, $x^{*}$ is a convex combination of $x^{1}$ and $x^{2}$. Thus, $x^{*}$ is not an extreme point solution. Contradiction.

Lemma $16 A^{1} \cup \cdots \cup A^{4}=\emptyset$.

Proof. We first assume that $\left|A^{1} \cup \cdots \cup A^{4}\right| \geq 2$. Suppose that $q, q^{\prime} \in A^{1} \cup \cdots \cup A^{4}$. Then, there are $4^{2}=16$ cases depending on which of the four sets $q$ and $q^{\prime}$ belong to.

Suppose that $q \in A^{1}$ and $q^{\prime} \in A^{2}$. Let $\epsilon>0$ be suitably small. Construct a new solution $x^{1}$ from $x^{*}$ by transferring $\epsilon$ from median $q$ to median $q^{\prime}+d$, i.e.

$$
x_{j j}^{\prime}= \begin{cases}x_{q q}^{*}-\epsilon, & j=q \\ x_{q^{\prime}+d, q^{\prime}+d}^{*}+\epsilon, & j=q^{\prime}+d \\ x_{j j}^{*}, & j \neq q, q^{\prime}+d\end{cases}
$$

and all other assignments are made according to (1). No component of $x^{*}$ is driven to zero by this change. Also, $x_{q^{\prime} q^{\prime}}^{*}+x_{q^{\prime}+d, q^{\prime}+d}^{*} \neq 1$, and from Lemma 15, $x_{q q}^{*}+x_{q+d, q+d}^{*} \neq 1$. Thus, no component of $x^{*}$ that is zero becomes non-zero. Further, from Lemma 14, no leaf node associated with an element of $A^{2}$ is part of any tight constraint (15). Thus, $x^{1}$ is feasible and satisfies all the same constraints that $x^{*}$ does.

Consider an alternative solution $x^{2}$ where $-\epsilon$ is transferred from median $q$ to median $q^{\prime}+d$ in $x^{*}$. Again, $x^{2}$ is a feasible solution. However, $x^{*}$ is a convex combination of $x^{1}$ and $x^{2}$. This contradicts the assumption that $x^{*}$ is an extreme point solution. 
The proof of the other fifteen cases are similar. When $q \in A^{1}$, the median value is transferred to and from vertex $q$. When $q \in A^{2} \cup A^{3} \cup A^{4}$, the median value is transferred to and from vertex $q+d$.

The above analysis analysis establishes that $\left|A^{1} \cup \cdots \cup A^{4}\right| \leq 1$. Now, suppose that $q \in A^{2}$. Since $1-\theta$ from vertex $c$ is assigned to the nearest median, let $q^{*} \in N_{c}$ such that $x_{c q^{*}}^{*}=1-\theta$. The $K$ median problem, ignoring constant terms, can be written as

$$
\begin{gathered}
\min f\left(\theta, x_{q+d, q+d}\right)=w_{c q^{*}}(1-\theta)+w_{q+d, q}\left(1-x_{q+d, q+d}\right)+\sum_{q \in A^{5}} 2 w_{q c} \theta \\
+\sum_{q \in A^{6} \cup A^{7}} w_{q c} \theta+\sum_{q \in A^{6} \cup A^{9}} w_{q+d, q}(1-\theta) . \\
\text { subject to } \begin{array}{r}
1 \geq \quad \theta \quad \geq 0 \\
\theta \geq x_{q+d, q+d} \geq 0 \\
\theta+x_{q+d, q+d}+(1-\theta)\left|A^{5}\right|+\left|A^{6}\right|+(2-\theta)\left|A^{7}\right|+\left|A^{8}\right| \\
+(1+\theta)\left|A^{9}\right|+2\left|A^{10}\right|=K .
\end{array}
\end{gathered}
$$

Observe that in this formulation, the constraints (15) are automatically satisfied and do not restrict the feasible set. In a linear program with two variables, upper bound, lower bound and one equality constraint, at an optimal extreme point one of the following must be true:

1. $\theta=0$, in which case the optimal solution is integral.

2. $\theta=1$, in which case the optimal solution is integral.

3. $x_{q+d, q+d}=0$, in which case $q+d \notin A^{2}$.

4. $x_{q+d, q+d}=\theta$, in which case $q+d \notin A^{2}$. 
Thus, $A^{2}=\emptyset$.

When $q \in A^{3} \cup A^{4}$, the proof is similar, with changes in the bounds of $x_{q+d, q+d}$.

When $q \in A^{1}$, the second variable in the linear program is $x_{q q}$. From Lemma 15, if $x_{q+d, c}^{*}=0$, then it remains 0 in the LP solution.

Theorem 14 Suppose equations (15) are included in $\left(L P_{2}\right)$. Then, the polytope is integral.

Proof. Because $A^{1} \cup \cdots \cup A^{4}=\emptyset$, the cardinality constraint $\sum_{j} x_{j j}=K$ is

$$
\begin{aligned}
K & =(1-\theta)\left|A^{5}\right|+\left|A^{6}\right|+(2-\theta)\left|A^{7}\right|+\left|A^{8}\right|+(1+\theta)\left|A^{9}\right|+2\left|A^{10}\right|+\theta \\
& =\left|A^{5}\right|+\left|A^{6}\right|+\left|A^{7}\right|+\left|A^{8}\right|+\left|A^{9}\right|+\left|A^{10}\right|+\left|A^{7}\right|+\left|A^{10}\right|+\theta+\left(\left|A^{9}\right|-\left|A^{5}\right|-\left|A^{7}\right|\right) \theta .
\end{aligned}
$$

Since $\left|A^{5}\right|+\left|A^{6}\right|+\left|A^{7}\right|+\left|A^{8}\right|+\left|A^{9}\right|+\left|A^{10}\right|=d$,

$$
K=d+\theta+\left|A^{7}\right|+\left|A^{10}\right|+\left(\left|A^{9}-\right| A^{5}|-| A^{7} \mid\right) \theta .
$$

Because all leaf nodes have median values $0, \theta$ or 1 , constraint (15) establishes that $\left|A^{7} \cup A^{10}\right| \leq K-d$. Thus,

$$
\begin{aligned}
K & \leq \theta+d+K-d+\left(\left|A^{9}\right|-\left|A^{5}\right|-\left|A^{7}\right|\right) \theta \\
\left|A^{5}\right|+\left|A^{7}\right| & \leq\left|A^{9}\right|+1
\end{aligned}
$$

Suppose that $\left|A^{5}\right|+\left|A^{7}\right|=\left|A^{9}\right|+1$. Then $\left\{j \in V: x_{j j}^{*}=1-\theta\right\}=\left\{j \in V: x_{j j}^{*}=\theta\right\}$. Transferring $\epsilon$ between those medians with value $\theta$ and those with value $1-\theta$ shows that $x^{*}$ is not an extreme point solution.

This contradiction implies that $\left|A^{5}\right|+\left|A^{7}\right| \leq\left|A^{9}\right|$ and that $\left|A^{7} \cup A^{10}\right|<K-d$. The first inequality shows that all constraints (15) are loose.

When $\left|A^{5}\right|+\left|A^{7}\right|=\left|A^{9}\right|$, there is one more median with value $\theta$, than value $1-\theta$. As a result, it is not possible to have a total median value of $K$.

Thus, we assume that $\left|A^{5}\right|+\left|A^{7}\right| \leq\left|A^{9}\right|-1$. Suppose that $\left|A^{9}\right| \geq 2$. Then, transfer $\epsilon$ between the leaf nodes associated with $A^{9}$. Because all constraints (15) are loose, we have that $x^{*}$ is not an extreme point solution. 
This contradiction establishes that $\left|A^{9}\right| \leq 1$ and that $A^{5} \cup A^{7}=\emptyset$. When $\left|A^{9}\right|=1$, then let $q \in A^{9}$. Transfer $\epsilon$ between $c$ and $q+d$ and between the vertices of each branch associated with $A^{6}$. This establishes that $x^{*}$ is not an extreme point solution.

Hence, $A^{9}=\emptyset$. The only remaining fractional vertices are $c$ and those associated with $A^{6}$. Because it is not possible to have a total median value of $K, c$ cannot be fractional.

\section{Some Open Questions}

To find the integer polytope, one important open question is how to characterize the set of constraints that describe the tree distances. Another interesting issue is that few results are known for the K-median problem on a tree with unit edge length. The graph given in Figure 2 has integral optimal point solutions for these arc distances. For the formulation of the 2-star, the inequalities (15) are exponential in the input size. The question of whether there is a linear size formulation is open. Finally, for which classes of graphs other than 2-stars, are the inequalities (10) useful in describing an integral polytope.

\section{Acknowledgments}

The research of the first author was supported in part by the Deutsche Forschungsgemeinschaft through the "Graduiertenkolleg Mathematische Optimierung" at the Universität Trier and in part by the German Federal Department of Education, Science, Research and Technology grant No. GR7TM1/02.05. The research of the third author was supported by Dean's Summer Fellowship from the Fisher College of Business of the Ohio State University. 


\section{References}

[1] V. Arya, N. Garg, R. Khandekar, V. Pandit, A. Meyerson and K. Munagala. 2001. Local search heuristics for $K$-median and facility location problems, 33st Annual ACM Symposium on Theory of Computing, 21-29.

[2] P. Avella and A. Sassano. 2001. On the p-median polytope, Mathematical Programming, 89, 395-411.

[3] I. Barany, J. Edmonds and L. Wolsey. 1986. Packing and Covering a Tree by Subtrees, Combinatorica, vol. 6, 221-233.

[4] 1983. D. C. Cho, E. L. Johnson and M. W. Padberg. On the uncapacitated plant location problem.I: valid inequalities and facets, Mathematics of Operations Research, 8, 579-589.

[5] G. Cornuejols and J. M. Thizy. 1982. Some facets of the simple plant location problem, Mathematical Programming, 23, 50-74.

[6] M. Goemans. 1992. Notes on the Median Polytope on Trees, notes.

[7] A. Kolen and A. Tamir. 1990. Covering Problems, in Discrete Location Theory, P. B. Mirchandani and R. L. Francis eds., Wiley-Interscience, New York, 263-304.

[8] P. Lemke. 1986. Some Properties of the $K$-median Problem Polytope, Ph.D. dissertation, Rensselaer Polytechnic Institute, Troy, NY.

[9] N. Megiddo, A. Tamir, E. Zemel and R. Chandrasekaran. 1981. An $O\left(n \log ^{2} n\right)$ algorithm for the $K$-th longest path in a tree with applications to location problems, SIAM J. on Computing, 10, 328-337. 
[10] P. B. Mirchandani. 1990. The p-median Problem and Generalizations, in Discrete Location Theory, P. B. Mirchandani and R. L. Francis eds., Wiley-Interscience, New York, 55-110.

[11] A. Tamir. 1996. An $O\left(p n^{2}\right)$ Algorithm for the $p$-median and related Problems on Tree Graphs, OR Letters, 19, 59-64.

[12] J. Ward, R. T. Wong, P. Lemke and A. Oudjit. 1994. Properties of the Tree K-median Linear Programming Relaxation, manuscript. 